la perte de matériaux nutritifs par suite des fermentations, exprimée en unités fourragères de l'aliment initial, ne dépasse pas sensiblement $10 \%$

$3^{\circ} \mathrm{La}$ distribution d'ensilage aux vaches laitières pendant la période critique d'alimentation d'été constitue une excellente pratique parce qu'elle permet d'éviter l'influence du régime de sous-nutrition, qui s'observe presque toujours à pareille époque sur les vaches bonnes laitières. Cette action favorable est particulièrement nette sur les vaches fraîchement vêlées.

- $\quad 4^{\circ}$ Le coût de l'ensilage de mais, compte tenu des frais élevés d'amortissement et d'entretien du silo, apparaît comms inférieur à celui des betteraves $1 / 2$ sucrières, à valeur fourragère égale bien entendu.

\title{
ANNEXE
}

Détermination des frais d'amortissement d'un silo métallique.

Le prix d'achat et les frais de montage d'un silo métallique de 140 mètres cubes pouvant contenir 100 tonnes d'ensilage est de 18.000 francs. Il est prudent d'amortir eette dépense en 15 ans. Si l'on admet un intérêt de $6 \%$ l'application de la règle d'aliments composés aboutit au paiement d'une prime annuelle de 1.915 francs.

Quant à la machine à ensiler, qui vaut 6.500 francs, il est logique de l'amortir plus rapidement, par exemple en cinq ans. Il faut pour cela une nouvelle prime annuelle de 1.875 francs.

Enfin, le coût der réparations de la machine et celui de l'entretien du silo (peinture avec enduit spécial, etc.) peuvent s'évaluer à 250 francs.

$\mathrm{Au}$ total, la dépense d'amortissement et d'entretien est donc de $1.915+1.875+250=4.040$ francs.

On peut se servir du silo pour faire d'abord un ensilage de vesce, destiné à être consommé pendant l'été, et correspondant à 50 tonnes d'herbe verte. Puis, ce fourrage consommé, on peut emmagasiner, à la fin du mois de septembre, 100 tonnes de maïs. Le silo sert donc pour la conserve de 150 tonnes d'herbe verte. Les frais d'amortissement rapportés à la tonne de fourrage vert sont, dans ces conditions, de 4.040

$$
\underline{-}=27 \mathrm{fr} \text {. }
$$

150

C'est ce prix de 27 franes qui a été utilisé dans le caleul de la dépense résultant de l'utilisation du maïs.

(A suivre)

\section{GERBER OU BABCOCK ?}

\author{
par le Dr Georges ROEDER
}

Dans le $\mathrm{n}^{0} 58$ de la Revue Le Lait, M. Em. Pozzi-Escot, professeur à l'Institut pour l'économie nationale du Pérou, membre de la American 
Chemical Society et de la Society of Chemical Industry, publie un article, dans lequel il attaque vivement le procédé GERBER pour la détermination de la teneur du lait en graisse et souhaite de le voir remplacer par le procédé BABCOCK.

Dans cet article qui, en maints d'endroits, est dénué d'objectivité scientifique, il prétend, sans du reste en apporter la preuve, que le procédé BABCOcK est de loin supérieur au procédé GERBER; et même qu'il présente par rapport à ce dernier les avantages suivants :

1) Simplicité dans le mesurage du lait;

simplicité dans les réactifs ;

simplicité dans le mode d'échauffement;

simplicité dans l'agitation et la centrifugation;

simplicité dans la lecture;

2) Mélange sans danger du lait avec l'acide sulfurique, car il n'y a pas lieu de secouer;

3) Pas d'échee par suite de départ de bouchons.

4) Impossibilité d'avoir des grumeaux de caséine dans le tube gradué;

a) car le lait est lavé par l'acide;

b) parce que le mélange est si simple, que rien ne peut être projeté dans le tube gradué ;

5) Donnée claire des résultats en poids \%, ce qui n'est pas le cas pour le procédé GERBER;

6) Exécution du dosage dans des conditions exactement connues, officiellement réglées; durée et vitesse de la centrifugation prescrites;

7) Connaissance parfaite des réactions et par suite possibilité de vérification sur la base même des données de BABCock.

Pour pouvoir établir une comparaison objective des deux méthodes, on a opposé, dans le tableau comparatif suivant, l'exécution du dosage d'après les deux procédés :

\section{GERBER}

10 a) Introduction de $10 \mathrm{~cm}^{3}$ de $\mathrm{SO}^{4} \mathrm{H}^{2}$ dans le butyromètre (au moyen d'une pipette ou d'appareils automatiques).

b) Addition de $11 \mathrm{~cm}^{3}$ de lait (au moyen d'une pipette).

c) Addition de $1 \mathrm{~cm}^{3}$ d'alcool amylique (au moyen d'une pipette ou d'appareils automatiques).

$2^{\circ}$ Mélange des substances introduites dâns le butyromètre fermé (bien agiter).

\section{BABCOCK}

$1^{\circ}$ a) Introduction de $17,5 \mathrm{~cm}^{3}$ de lait dans le flacon (au moyen d'une pipette).

b) Addition de $17,5 \mathrm{~cm}^{3} \mathrm{SO}^{4} \mathrm{H}^{2}$ (au moyen d'un verre gradué).

$2^{\circ}$ Mélanger les substances introduites dans le flacon ouvert en provoquant un mouvement circulaire. 
$3^{\circ}$ a) Placer dans un bain-marie à $65-70^{\circ} \mathrm{C}$. (seulement dans le cas où il y a plusieurs échantillons).

b) Introduction des butyromètres dans la centrifugeuse.

$4^{\circ}$ centrifuger: $3-5$ minutes à 800-1.000 tours.

$5^{\circ}$ Retirer et lire (dans le cas de plusieurs échantillons seulement, chauffer encore à $65-70^{\circ}$ C.).

Lecture :

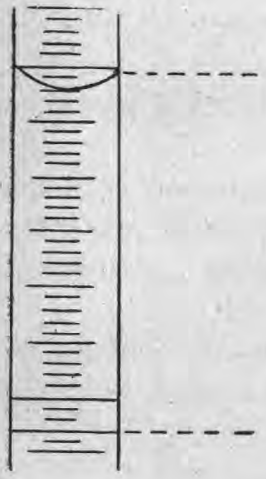
Ménisque infé- rieur, graisse limpide, tige plate, divisée en $1 / 10 \%$, à environ $65^{0}$.

Surface de séparation plane que l'on peut faire affleurer à une division à volonté. $3^{\circ}$ a) Introduction des flacons dans la centrifugeuse.

b) Chauffer la centrifugeuse avec de l'eau bouillante ou de la vapeur (constamment).

-40 Centrifuger : 6-7 minutes à 700-1.200 tours.

$5^{\circ}$ Arrêter la centrifugeuse, l'ouvrir et remplir les flacons avec de l'eau bouillante.

$6^{\circ}$ Centrifuger encore une fois (1 minute).

$7^{\circ}$ Retirer et lire.

$8^{\circ}$ a) Si la graisse n'est pas claire, chauffer encore dans la centrifugeuse en tournant lentement.*

b) Retirer à nouveau et lire.

\section{Lecture :}

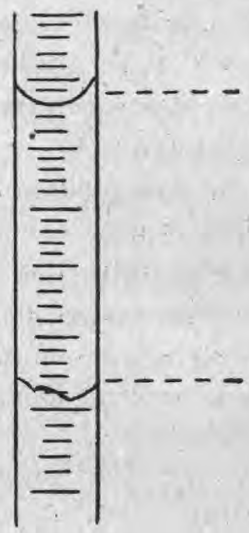

Ménisque supérieur, graisse fréquemment trouble, tige ronde. divisée en $1 / 5 \%$, à environ $95^{\circ}$.

Surface de séparation irrégulière qui tombe rapidement et ne peut être corrigée.

Nous laissons au lecteur le soin de se faire lui-même une opinion d'après la confrontation claire et serrée qui précède; s'il la considère en quelque sorte objectivement, cette opinion ne manquera pas d'être en faveur du procédé GERBER. Il faut constater - à l'encontre des déductions du prof. Pozzi-Escot - que :

* Barthel, Methoden zur Untersuchung von Milch und Molkereiprodukten, III. Edition 1920, page 56, écrit à ce propos: Si la graisse apparait trouble, il faut remettre les flacons à chauffer dans la centrifugeuse tournant lentement; la graisse devient alors assez claire le plus souvent. is 
1) La méthode GERBER est de loin nettement supérieure en simplicité, aussi bien en ce qui concerne l'appareillage que l'exéeution du. dosage.

\section{A) Appareillage:}

a) On n'a besoin que d'une centrifugeuse simple, dont les frais d'acquisition et de fonctionnement sont peu élevés, tandis que pour la méthode BABCOCK une centrifugeuse chauffable, et même autant que possible une centrifugeuse à vapeur, est indispensable, si l'on veut, au moins dans la majorité des recherches, obtenir une séparation à peu près claire de la graisse, et des résultats auxquels on puisse se fier. Nous savons par des notices bibliographiques sur la détermination de la graisse en Amérique - pays d'origine de la méthode BABCOCK —, que, malgré que cette nécessité soit reconnue, l'acquisition et l'exploitation d'une centrifugeuse chauffable sont pratiquement impossibles, dans de très nombreux cas. Cette dépendance d'un moyen de secours, qui souvent ne peut être employé qu'avec de grandes difficultés, souvent même ne peut pas l'être du tout, je la considère comme un désavantage essentiel du procédé BABCock, et je suis en cela - autant que je sache - d'accord avec des opinions fréquemment exprimées.

b) La forme en apparence plus simple, ou plutôt plus pratique, des flacons de BABCOCK, entraîne tant de désavantages par rapport aux butyromètres GERBER (voir plus bas) qu'à mon avis, on peut en recommander l'élimination.

\section{B) Exécution :}

aa) L'introduction des réactifs, généralement au moyen de pipettes ou d'appareils mesureurs construits exprès, qui sont encore plus faciles à manier que les pipettes, s'effectue par l'ouverture large du butyromètre GERBER, rapidement, facilement et peut-être faite par quelqu'un qui n'a pas de connaissances préalables ou qui n'a pas l'habitude des travaux analytiques; 'il est impossible de verser du liquide à côté, ete.

L'introduction de la pipette à lait par l'étroit tube gradué des flacons de BABCOCK est déjà nettement plus difficile pour le profane; et eeci est encore plus vrai pour l'acide sulfurique que l'on verse avee un récipient gradué et pour le lavage quantitatif(!) de la partie graduée du col. Il faut prendre garde, en même temps, que l'acide sulfurique, sans se mélanger au lait, se dispose par couches au-dessous de celui-ci et qu'aucun acide ne coule hors du col et ne cause quelque dommage. (Pour exécuter réellement proprement et exactement cette opération, il faut déjà y employer des gens qui ont travaillé quelques semestres dans un laboratoire d'Ecole supérieure technique, mais pas de praticiens).

bb). On ne voit pas à quel égard le travail avec des récipients fermés 
constituerait un désavantage par rapport à celui que l'on réalise avec les flacons de BABCOCK ouverts.

cc) L'échauffement du mélange lait-acide s'obtient déjà de lui-même à la température désirée, à la condition qu'il y ait seulement quelques échantillons à étudier, c'est-à-dire lorsque le mesurage, le mélange et le passage dans la centrifugeuse peuvent se succéder rapidement. S'il y a plusieurs échantillons à examiner, les flacons fermés - sans manipulation complémentaire avec leur contenu - n'ont qu'à être placés dans un bain-marie de 60 à $70^{\circ} \mathrm{C}$.

D'après BABCock, au contraire, la centrifugeuse doit être chauffée à la température d'ébullition au moyen d'eau bouillante ou de vapeur; et les flacons, après qu'ils ont déjà été soumis une première fois à la centrifugation, doivent être encore une fois remplis d'eau bouillante, pour passer à nouveau dans la centrifugeuse chauffée. Il n'est pas encore certain alors que la graisse soit suffisamment claire pour pouvoir être lue ; si ce n'est pas le cas, les flacons doivent à nouveau être placés dans la centrifugeuse chauffée et être encore une fois soumis au chauffage en même temps qu'à une lente rotation.

(C'est ce que le professeur Pozzi-Escor appelle: "Plus grande simplicité du procédé BABcooK dans le chauffage et dans la centrifugation n).

Qu'il me soit cependant permis d'insinuer ici la question de savoir si vraiment ce procédé pourtant un peu long est exactement observé dans la pratique? Je crois que, dans de nombreux cas, on se contentera, aux dépens de la justesse du résultat, de centrifuger une fois, deux fois au plus, pour lire ensuite, que la graisse apparaisse alors plus ou moins trouble et quitte à obtenir ainsi des résultats légèrement supérieurs ou inférieurs. Mais la certitude accordée à toute la méthode pourrait en souffrir fortement; danger qui est impossible avec le procédé Gerber, car ici - exception faite des cas exceptionnels - on obtient une séparation nette de la graisse et une lecture précise avec un seul passage à la centrifugeuse.

dd) La lecture, elle aussi, est, d'après le procédé GERBER, plus simple, plus certaine et plus exacte ; et cela pour les raisons suivantes :

a) Le niveau de séparation entre la colonne de graisse et la solution située en dessous est parfaitement plan dans le procédé GERBER, tandis qu'il ne l'est pas dans le procédé BABcock.

En appuyant légèrement sur le bouchon ou en le retirant un peu, on amène facilement la surface de séparation sur un trait de division, ce qui facilite beaucoup la lecture.

Dans les flacons de BABCOCK, par contre, à la lecture déjà difficile en soi de la surface inférieure de séparation, s'ajoute encore cette nouvelle difficulté qu'une correction de ce genre n'est pas possible au moyen d'une fixation volontaire du point de départ de la colonne de graisse. 
ß) La lecture s'effectue à environ $65^{\circ}$, ainsi à une température sensiblement plus basse que suivant le procédé BABCock. Aussi la différence de température par rapport à la température environnante estelle plus réduite: l'équilibre de température, e'est-à-dire le refroidissement et la contraction correspondante du contenu des flacons (aussi bien la graisse que la solution située au-dessous) s'effectue beaucoup plus lentement. Il en résulte que l'on dispose de plus de temps pour la lecture, e'est pourquoi elle peut être faite avec plus de soin et plus d'exactitude. On peut encore s'opposer à l'abaissement de la colonne de graisse en appuyant sur le bouchon de caoutchoue (voir plus haut), tandis que dans les flacons de BABCOCK, la colonne de graisse est constamment en mouvement pendant la lecture.

ү) La graisse est parfaitement claire et constitue des ménisques extraordinairement nets, tout particulièrement dans les butyromètres à tige plate dont l'utilisation aujourd'hui généralisée a été facilitée par ce fait que l'on n'a pas besoin d'introduire les liquides par le tube gradué (comme cela doit se faire dans le cas des flacons de BABCocK).

ઈ) Cette dernière circonstance permettait également, par suite du rétrécissement devenu ainsi possible des tubes gradués une division de l'échelle nettement plus avantageuse, que j'oppose sans autres commentaires à la division de BABCOCK :

\begin{tabular}{|c|c|}
\hline GERBER & BABCOCK \\
\hline $\begin{array}{l}\text { Longueur de l'échelle : } 6,5-7 \mathrm{~cm} \text {, } \\
\text { soit : } \\
6 \text { ou } 8 \% \text { divisé en } 1 / 10 \% \text {; } \\
1 / 10 \%=0,8-1.1 \mathrm{~mm} \text {. } \\
0,05 \% \text { sont appréciés avec certitude } \\
\text { entre deux traits de graduation. }\end{array}$ & $\begin{array}{l}\text { Longueur de l'échelle : } 5,5-6 \mathrm{~cm} \text {., } \\
\text { soit : } \\
10 \% \text {, divisé à } 1 / 5 \% \text {; } \\
1 / 5 \%=0,9-1,2 \mathrm{~mm} \text {. } \\
0,1 \% \text { peut encore être apprécié. } \\
0,05 \% \text { ne peut plus être apprécié ou } \\
\text { du moins ne peut plus l'être avee cer- } \\
\text { titude. }\end{array}$ \\
\hline
\end{tabular}

2) En ce qui concerne ce qu'il y a de dangereux dans ces méthodes il est complètement impossible, si l'on opère d'après les prescriptions du procédé Gerber, de se blesser par suite d'une projection de bouchon ou par suite d'une projection d'acide sulfurique. Ce serait plutôt dans le cas de la méthode BABCock qu'il y aurait danger de s'échauder du fait d'un travail continuel avec de l'eau bouillante et de la vapeur chaude.

D'ailleurs, si M. le professeur Pozzr-Escot peint avec des couleurs suggestives ce qu'il vit, alors qu'il entrait pour la première fois dans son laboratoire d'enseignement: un élève occupé à la détermination de Gerber se blessa avec de l'acide sulfurique par suite de l'éclatement d'un bouchon, et puisque cet événement fit sur lui une impression " décisive ", je puis lui opposer en toute eonscience ce qui se passa lors de ma première expérience d'après le procédé BABCOCK : malgré un travail prudent, une des petites fioles a parois minces éclata dans la 
centrifugeuse et, si je ne fus pas blessé, le manteau d'une collaboratrice placée non loin de là fut complètement inondé d'acide sulfurique. Si ce n'était pour répondre aux reproches incessants de M. le professeur Pozzi-Escot, j'aurais tenu l'aceident pour un hasard malheureux trop insignifiant pour en dire seulement un mot.

3) Ce qui a été déduit au sujet de ce qu'il a prétendu être dangereux dans le procédé GERBER, est également vrai pour l'insuccès d'expériences, ear le professeur Pozzi-Escot veut ramener l'un et l'autre à la même cause (projection de bouchons).

4) La possibilité d'une persistance de particules de caséine dans le tube gradué, doit, à mon avis, être négligée. Comme M. le professeur Pozzi-Escot le consigne dans un autre passage de son article, la caséine; après qu'elle a précipité, est dissoute par l'acide. Dans le cas d'une vitesse angulaire de 1.000 tours par minute, avec un diamètre du plateau de $15-25 \mathrm{~cm}$., (correspondant à la longueur des tubes gradués), cela correspond à une vitesse périphérique de $11 \mathrm{~m}$. par seconde et je crois qu'il est impossible que des parties de la solution restent encore adhérents aux tubes gradués.

Par contre, je erois que, dans le eas de la méthode GERBER, les derniers restes de la graisse se laissent plus facilement, avec le secours de la force centrifuge, séparer de la solution à forte teneur en $\mathrm{SO}^{4} \mathrm{H}^{2}$, et d'un poids spécifique trés élevé, que, dans la méthode BABCocK, de la solution fortement étendue d'eau ultérieurement, et par conséquent spécifiquement plus légère. (Il y a peut-être là une relation avec le trouble de la graisse séparée, dans le procédé BABCock?)

5) Il est bien entendu que pour nos expériences la donnée de la teneur en graisse s'exprime en pour cent du poids de lait (gr. dans 100 gr.). Le volume lu et employé a été converti convenablement lors du jaugeage des instruments - exactement de la même façon que BABCock faisait cette opération. Ainsi la méthode $\mathrm{BABCOCK}$, qui permet aussi bien de mesurer le volume de lait que de lire le volume de graisse, n'offre pas plus et pas moins d'occasions d'erreurs que le procédé GERBER, dans les pays qui donnent la teneur en graisse en grammes par litre.

6) Si le professeur Pozzi-Escot voit en outre une supériorité de la méthode BABCOCK, dans le fait que pour celle-ci la marche de l'expérience est exactement fixée, il faut lui répondre que ceci a eu lieu d'une manière exactement semblable dans le procédé GERBER - y compris la fixation de la vitesse et.la durée de la centrifugation, ce que le professeur Pozzi-Escot met particulièrement en évidence dans son article (voir plus haut).

Que la conduite du procédé GERBER puisse apparaître, en gros, peut-être un peu plus simple, la raison en est seulement que justement la méthode dans son ensemble est effectivement plus simple. Il n'existe 
d'ailleurs pas pour cela de règlement légal (1); mais, à mon avis, jl ne faut attribuer à cette circonstance, le cas échéant, aucune signification pratique. Nous n'avons aussi pas de règlement officiel pour d'autres méthodes de détermination; celui qui veut obtenir des résultats certains, doit effectuer ses expériences exactement et selon les prescriptions du cas. Celui qui le néglige et travaille sans soin obtient des résultats inexacts - avec ou sans ratification officielle.

7) En ce qui concerne, enfin, la parfaite connaissance des processus (et le sentiment personnel de sûreté dans l'exécution des opérations), je me permets d'affirmer que la connaissance exacte des processus est, dans le cas du procédé BABCOCK, aussi peu parfaite quế dans celui du procédé GERBER.

Il est prouvé que la graisse séparée dans le procédé GERBER concorde exactement, en volume, avee la quantité de graisse contenue dans le lait: cela a été établi par des travaux expérimentaux approfondis et confirmé, le plus exactement possible par d'innombrables déterminations comparées avec les procédés de l'analyse pondérable. Que la graisse du butyromètre soit identique à la graisse originaire du lait ou qu'elle soit modifiée pour une petite part et ait été remplacée par d'autres substances, cela reste sans importance pour le résultat.

II semble douteux que la graisse du lait en contact avec l'acide sulfurique très concentré, à la température d'ébullition de l'eau, comme il est prescrit dans le cas du procédé BABcook, ne subisse pas une altération. En tout cas, je n'ai pas connaissance qu'il ait été prouvé, que, dans le procédé BABCock la graisse ne s'altérait pas. En concluant d'après les mesures du procédé BABCOCK, on devrait croire cependant qu'il se présente une modification de la graisse. Le tube gradué contient à $15^{\circ} \mathrm{C}$. de $0-10 \%: 2,00 \mathrm{~cm}^{3}$. Après que $17,5 \mathrm{~cm}^{3}$, qui équivalent à $18 \mathrm{gr}$. de lait ont été utilisés, l'histoire de l'établissement de cette échelle se pose d'elle-même : On a adopté 0,9 comme poids spécifique de la graisse, d'où $1,8 \mathrm{~g}$. ( = $10 \%$ ) de graisse occupent un volume de $2 \mathrm{~cm}^{3}$.

Mais les graisses liquides chauffées se dilatent considérablement, tandis que le verre ne possède qu'un coefficient de dilatation très réduit (environ 0,000.008, dilatation linéaire). Ainsi le volume de l'échelle n'est augmenté, pendant le chauffage, que d'une façon tout à fait insignifiante, tandis que le volume de la graisse subit un accroissement sensible. On aurait donc dû lire des résultats beaucoup trop élevés, ce qui - nous ne discuterons pas comment - n'est pas le cas

(1) D'après la traduction que j'ai sous les yeux et comme je l'apprends par ailleurs, il ne doit du reste pas s'agir en Amérique d'un réglement officiel dans le sens strict du mot, mais d'un accord officiel entre les milieux spécialisés intéressés.

Mais la question d'un tel processus ne pét être envisagée pour la méthode GERBER, d'un usage général ici, car dès le début la détermination est fixée exactement dans toutes ses parties et il est impossible de s'en écarter. 
d'après l'expérience et d'après la concordance existant entre les résultats et des dosages parallèles effectués par l'analyse pondérable.

A mon avis, on peut l'expliquer en disant que toute la graisse ne se sépare pas sans modification, et qu'à la place du déficit qui se produit, a lieu un accroissement de volume de la graisse séparée. Mais ce processus dépend de l'influence de toutes sortes de facteurs (par exemple, sans aller plus loin, la composition inconstante de la graisse du lait) et c'est pourquoi cela semble, même malgré un règlement officiel, invérifiable. Et la perfection de notre connaissance des processus à envisager s'arrête là.

Comme criterium décisif pour l'exactitude des résultats, il reste la comparaison avec un procédé analytique reconnu comme procédétype. Mais, pour les deux méthodes, on a déjà la preuve d'une bonne concordance des résultats avec l'analyse pondérable. Il ne s'agit donc plus que de savoir laquelle des deux mérite la préférence en ce qui concerne les questions discutées. Enfin il faut encore considérer (et cela est vrai pour les deux procédés) que l'exàctitude de toutes les méthodes d'investigation analogues rencontre une limite naturelle. Les méthodes rapides pour le dosage de la graisse du lait (GERBER aussi bien que BАBCOCK), de même que des méthodes analytiques et comme beaucoup d'autres méthodes biochimiques de recherche, ne sont pas des méthodes d'analyse quantitative, telles que celles que nous pouvons employer pour le dosage des métaux, et dans lesquelles il est possible, une fois un corps anorganique obtenu, de le conserver à volonté pendant des heures, à l'incandescence, sans qu'il se modifie.

Nous avons plutôt affaire à des substances tout à fait instables qui se modifient facilement dans toutes les conditions possibles. C'est pour cela aussi que d'autres procédés très appréciés comme méthodes analytiques doivent être exécutés si strictement d'après des instructions précises (indice d'iode, indice de ReIchert-Meisst, etc.), qu'ici les dimensions des récipients, dans lesquels sont effectuées les recherches, jouent un rôle décisif. Dans toutes ces méthodes, on sait d'avance que l'on n'obtient pas un produit final immuable, mais que l'on peut trouver uniquement un chiffre obtenu d'une façon empirique et qui a un certain rapport avec les qualités à évaluer de la substance examinée.

Le dosage de la graisse, tout spécialement, a, sur différents autres dosages du même genre, l'avantage que, en général, ce que l'on obtient comme graisse, ou plutôt ce qui est considéré comme la graisse trouvée, approche assez de la teneur réelle en véritable graisse. Mais il ne faut pas oublier que la graisse elle-même (la graisse du lait ou la graisse du beurre) ne constitue pas un corps présentant de l'unité et exactement défini; on sait qu'il peut se modifier dans ses propriétés sous différentes influences, par exemple sous l'influence de circonstances alimentaires, et que ces modifications se feront sentir aussi bien dans ses utilisations pratiques que dans les examens qu'on pourra en faire. 
Par conséquent, dans chaque méthode, on ne peut prendre pour base que des valeurs moyennes qui peuvent présenter des écarts en plus ou en moins. L'exécution ultérieure de la méthode doit, de toute façon, avoir lieu d'une manière plus ou moins empirique, et les possibilités d'erreurs qui reposent sur les écarts primitifs de la base doivent être admises, qu'on le veuille ou non.

Mais à la question de savoir à laquelle de deux méthodes de ee genre on doit, dans la pratique, donner la préférence, il faudra, les résultats des deux méthodes étant également satisfaisants, choisir tout simplement la plus simple, surtout lorsqu'il s'agit d'une méthode tochnique rapide de dosage qui doit être exécutée par des non initiés.

Il n'est pas douteux que la méthode GERBER est certainement supérieure à la méthode ВАBCock sous le rapport de la simplieité ainsi que cela a été exposé, dans ce qui précède, d'une façon claire et détaillée.

\title{
LE MARCHÉ des GRUYÈRES ET LA NOUVELLE FABRICATION DU GRUYËRE EN BOITE
}

\author{
par M. L. ROY,
}

Directeur de l'Ecole Nationale d'industrie laitière de Mamirolle (Doubs)

La hausse du franc a provoqué une perturbation profonde dans nos industries nationales. L'agriculture et ses industries connexes sont aussi gravement atteintes et une légère mévente a frappé dernièrement la plus ancienne et la plus importante de celles-ci: la fabrication du gruyère. L'industrie fromagère de Franche-Comté et des Savoies a passé à l'autome par une crise qui, quoique peu grave, doit cependant nous préoccuper, d'autant plus qu'elle concerne la presque totalité de nos agriculteurs et qu'elle a failli mettre en péril même le principe coopératif de nos vieilles fruitières. La crise a été três rapidement limitée, grâce surtout à un fromage dérivé du gruyère, de belle présentation et d'exportation facile, d'appelations variables et fantaisistes qui n'est autre que de la pâte de gruyère.

Qu'est-ce que la pâte de gruyère et quel rôle peut-elle bien jouer au point de vue économique? Voilà deux questions que je vais essayer d'exposer.

La. pâte de gruyère, comme son nom l'indique, est un fromage gras, de qualité supérieure lorsqu'il est bien préparé, hygiénique et sain en raison de son processus de fabrication aujourd'hui entièrement mécanique, d'un poids et d'un format voisin du camembert et fabriqué avec des gruyères de bonne qualité, mais classés de deuxième ou troisième choix quant à leur ouverture.

Nul n'ignore qu'un gruyère peut être parfait de pâte et de goût et cependant ne pas être apprécié par la clientèle riche qui aime à voir 